\section{A case of idiopathic perifoveal telangiectasia preceded by features of cone dystrophy}

D Barthelmes, MC Gillies, JC Fleischhauer and FKP Sutter
Eye (2007) 21, 1542-1543; doi:10.1038/sj.eye.6703011

Correction to: Eye advance online publication, 21 September 2007; doi:10.1038/sj.eye.6702985

Owing to a publishing error, there is a discrepancy between the captions in Figures 1 and 2 and their respective legends. The correct figures and legends are shown below.

The typesetters would like to apologise for this mistake.
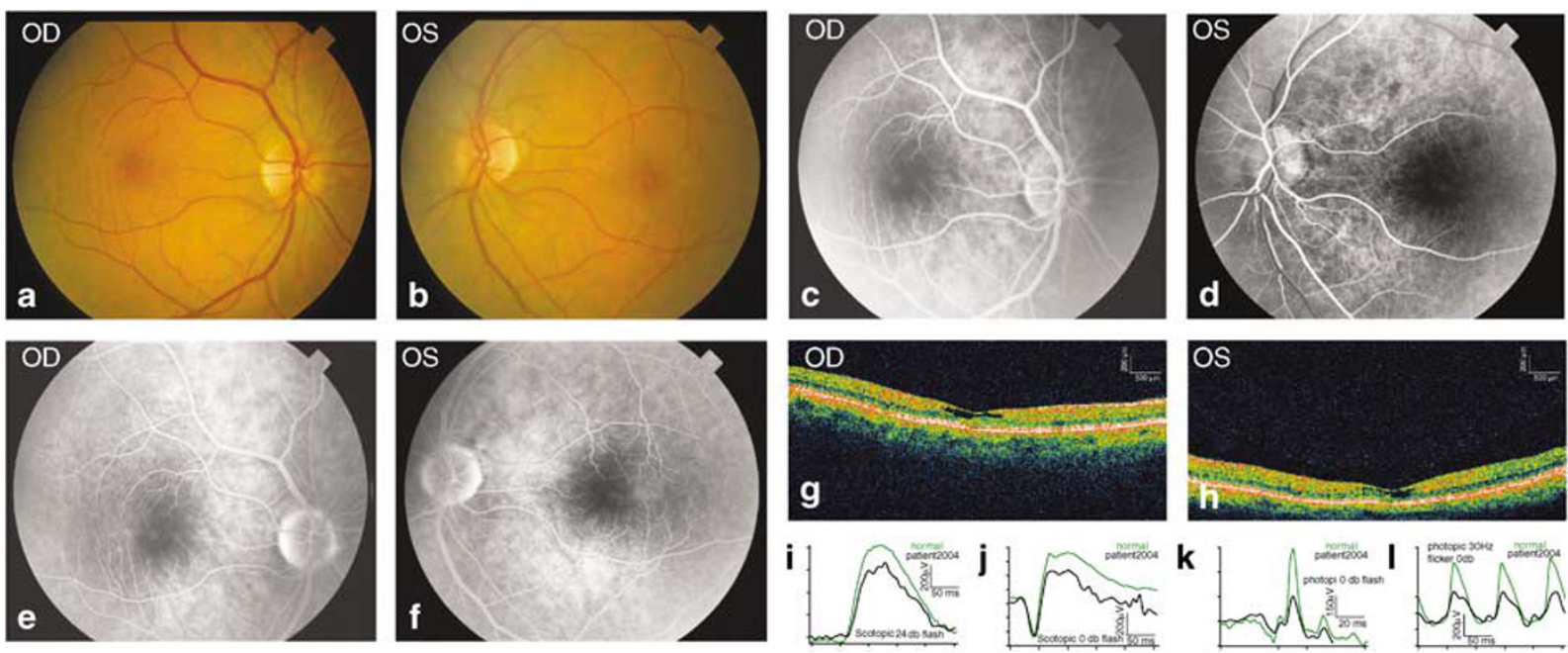

Figure 1 Right (a) and left eye (b) fundus photographs in 2004 show pigment changes in the central macula. Early fluorescein angiographs (c and d) reveal a central window defect. Late fluorescein angiography images (e and f) show irregularities in background fluorescence. No telangiectasis or vessel staining is visible. OCT images ( $\mathrm{g}$ and $\mathrm{h}$ ) exhibit foveal inner-lamellar holes. Ganzfeld-ERG recordings demonstrate normal rod-driven responses ( $\mathrm{i}$ and $\mathrm{j}$ ) and reduced amplitudes in cone-driven responses $(\mathrm{k}$ and $\mathrm{l}$ ). 

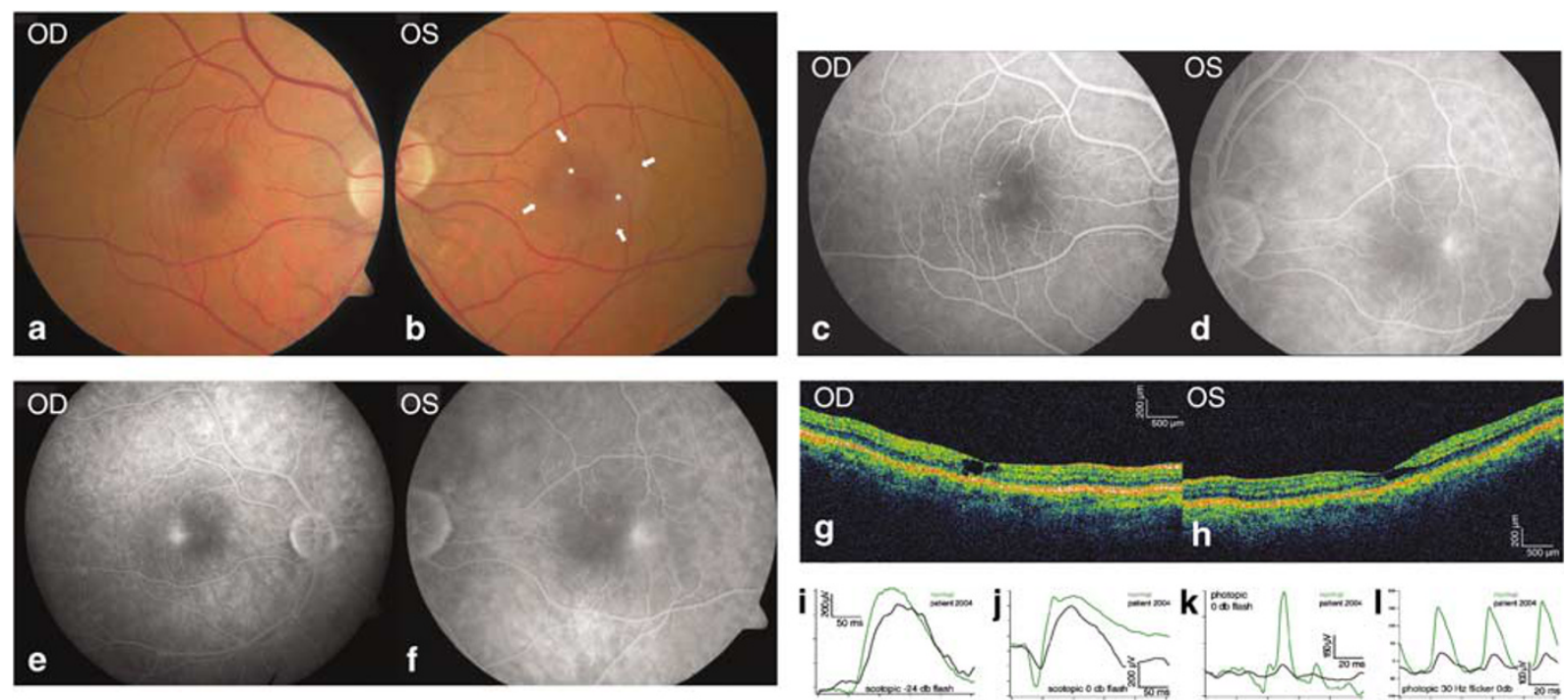

Figure 2 Fundus photographs taken in 2007 ( $a$ and b) show a loss of transparency (arrows) of the central macula and pigment abnormalities with right-angled vessels $\left(^{*}\right)$ and crystalline deposits in the temporal perifovea. Early fluorescein angiography images (c and d) show telangiectatic vessels in the temporal perifoveal zone, while late angiographs (e and $\mathrm{f}$ ) show more generalised hyperfluorescence. OCT images ( $\mathrm{g}$ and $\mathrm{h}$ ) still show slightly larger inner-lamellar holes. Rod-driven responses in Ganzfeld-ERG (i and j) are still normal while cone-driven responses have decreased further ( $k$ and $l)$. 